\title{
BMJ Open Quality Making surgical wards safer for patients with diabetes: reducing hypoglycaemia and insulin errors
}

\author{
Aminder Singh, Angela Adams, Bethany Dudley, Eliza Davison, Lauren Jones, \\ Lucy Wales
}

To cite: Singh A, Adams A, Dudley $B$, et al. Making surgical wards safer for patients with diabetes: reducing hypoglycaemia and insulin errors.BMJ Open Quality 2018;7:e000312. doi:10.1136/ bmjoq-2017-000312

Received 28 December 2017 Revised 1 July 2018 Accepted 5 July 2018
Check for updates

(C) Author(s) (or their employer(s)) 2018. Re-use permitted under CC BY-NC. No commercial re-use. See rights and permissions. Published by BMJ.

Northern Vascular Centre, Newcastle Upon Tyne Hospitals NHS Foundation Trust, Newcastle Upon Tyne, UK

Correspondence to Dr Aminder Singh; as.09@hotmail.co.uk

\section{ABSTRACT}

High-quality perioperative diabetes care is essential to improve surgical outcomes for patients with diabetes. Inadequate perioperative diabetes care is associated with increased wound complications, higher mortality rates and increased length of hospital stay. Despite national guidelines, surgical wards remain a high-risk area for poor diabetes care. An initial baseline audit in 2014 of vascular patients with diabetes undergoing major lower limb amputation identified poor glycaemic control in 90\% of patients, with high rates of hypoglycaemia and insulin management errors in $75 \%$. Less than $15 \%$ of patients received specialist diabetes input and $20 \%$ required third-party assistance for hypoglycaemia. This quality improvement project aimed to reduce hypoglycaemia, insulin management errors and patient harm events by $50 \%$ in vascular surgery patients over a 3-year period. Key interventions over three successive Plan, Do, Study, Act cycles included educational and guideline initiatives (2015), establishing a diabetes in-reach service (2016) and implementing a whiteboard sugar cube alert system for poor glycaemic control (2017). The final introduction of the whiteboard sugar cube alert system delivered the greatest impact in reducing hypoglycaemia rates by more than $50 \%$, insulin management errors by $70 \%$ and patient harm events by $75 \%$.

\section{PROBLEM}

Diabetes mellitus is the the most common disorder of metabolism and is characterised by relative or absolute deficiency of endogenous insulin. Data from the 2017 National Diabetes Inpatient Audit (NaDIA) showed 1 in $15(7 \%)$ of the total population have diabetes and one in six hospital beds are occupied by a patient with diabetes. ${ }^{1}$ Diabetes predisposes the patient to an array of secondary microvascular and macrovascular complications and, through the pathological process of atherosclerosis, can lead to peripheral arterial disease. Severe peripheral arterial disease leads to critical limb ischaemia, which is an indication for lower limb amputation (LLA). Diabetes the leading cause of non-traumatic $\mathrm{LLA}^{2}$ with incidence of LLA 8-15 times higher in this group of patients. ${ }^{34}$
Despite nationwide recognition that highquality perioperative diabetes care improves surgical outcomes, people undergoing surgery often receive inadequate diabetes care. ${ }^{5}$ The National Confidential Enquiry into Patient Outcome and Death (NCEPOD, 2014) identified people with diabetes undergoing LLA as a high-risk group for hypoglycaemia, medication errors and inadequate specialist diabetes input. ${ }^{6}$

The Northern Vascular Centre (NVC, Freeman Hospital, Newcastle, UK) is a major tertiary referral centre for the North East of England, admitting more than 2000 vascular patients per year. A third of these patients are admitted as an emergency and $30 \%-40 \%$ have diabetes. To understand the challenges of delivering safe diabetes care to vascular surgery patients, the NVC audited standards of diabetes care in people undergoing major LLA over a 6-month period from January 2014. This baseline audit identified poor glycaemic control in $90 \%$ of patients, with insulin management errors in $75 \%$. Less than $15 \%$ of patients received specialist diabetes input, highlighting an urgent need for improvement.

While the project team understood that the busy surgical ward environment may have contributed to the problem, it was believed that systems and processes could be improved, which could ultimately benefit all vascular surgery inpatients with diabetes. The project aimed to reduce the prevalence of hypoglycaemia, insulin management errors and patient harm events in vascular surgery patients undergoing major-either below knee or above knee-LLA by $50 \%$ over a 3-year period.

\section{BACKGROUND}

The care of people with diabetes is complex, particularly for those undergoing surgical procedures. Inadequate perioperative diabetes control is associated with poor 
outcomes, including increased wound complications, higher mortality rates and increased length of hospital stay. ${ }^{7-9}$

The reasons for these adverse outcomes are multifactorial but include poor glycaemic control, medication errors, lack of institutional guidelines and inadequate knowledge of diabetes among staff delivering care. ${ }^{10} 11$ High-quality diabetes care on surgical wards is an essential component of the overall pathway of clinical care delivered to surgical patients with diabetes.

Despite national guidelines aiming to improve perioperative diabetes care, concerns have been raised as to how well guidelines are adhered to. National audits consistently report surgical patients are at higher risk from medication errors and hypoglycaemia. ${ }^{1}$ NCEPOD advised that the care of diabetes patients undergoing LLA was poor or unacceptable in $27 \%$ of patient pathways. ${ }^{6}$ Principal NCEPOD recommendations included increasing specialist nurse input, reducing medication errors and clear guidelines for the management of blood glucose levels. Perioperative management of surgical patients with diabetes is the subject of a national ongoing NCEPOD enquiry due for completion in 2018.

\section{STANDARDS}

The NaDIA definitions for deranged capillary blood glucose were used with mild hypoglycaemia defined as levels $3-3.9 \mathrm{mmol} / \mathrm{L}$, severe hypoglycaemia as readings $<3 \mathrm{mmol} / \mathrm{L}$, hyperglycaemia as levels $>12 \mathrm{mmol} / \mathrm{L}$ and persistent hyperglycaemia as blood glucose $>12 \mathrm{mmol} / \mathrm{L}$ on two consecutive days. ${ }^{1}$ Diabetes monitoring was considered adequate if capillary blood glucose measurements were recorded once daily for diet-controlled diabetics, twice daily for oral hypoglycaemic agent-controlled diabetics and four times daily for those on insulin. ${ }^{1}$ Satisfactory glycaemic control was described as no blood glucose reading $<4 \mathrm{mmol} / \mathrm{L}$ and no more than one reading $>12 \mathrm{mmol} / \mathrm{L}$ in a 24-hour period. ${ }^{1}$ A patient had poor control if there was a blood glucose reading $<4 \mathrm{mmol} / \mathrm{L}$ or more than two readings $>12 \mathrm{mmol} / \mathrm{L}$ in a 24 -hour period. ${ }^{1}$ Specialist input was defined as clinical review of a diabetes patient by a diabetologist or the diabetes specialist nurse and the NCEPOD recommendations that all patients undergoing LLA should receive specialist review within 12 hours of admission as well as both preoperatively and postoperatively were used. ${ }^{6}$ A medication error was defined as absence of appropriate change to, or documented review of, medication regime after a hypoglycaemic or persistent hyperglycaemic event. ${ }^{6}$ A patient harm event was defined as injectable medical therapy being required as a consequence of inadequate diabetes control.

\section{BASELINE MEASUREMENT}

Data were collected over a 6-month period (2014) for consecutive patients with diabetes undergoing major LLA.
Over 6 months, 751 inpatient diabetes days were evaluated. Poor glycaemic control was evident in 293 (39\%) days and $80 \%$ of patients were exposed to medication errors. Preoperative and postoperative specialist input was received by $15 \%$ of patients and $20 \%$ of patients suffered patient harm events, requiring third-party assistance for hypoglycaemia. It was clear from this data collection that the quality of diabetes care needed to be improved.

\section{DESIGN}

The project's aim was to reduce the prevalence of hypoglycaemia, insulin management errors and patient harm events in vascular surgery patients by $50 \%$ over a 3-year period (2015-2017). This figure was agreed at the 'Sign Up to Safety' diabetes working group meeting by a multidisciplinary approach, involving key stakeholders including a consultant diabetologist, senior ward sister, consultant vascular surgeon, diabetes specialist nurse, together with rolling representatives from ward medical and nursing staff.

We sampled patients undergoing LLA as a high-risk group with predictable extended lengths of hospital stay, enabling effective evaluation of day-to-day standards of diabetes care on the vascular surgery ward. Appropriate patients were identified from the hospital vascular activity database and data were collected from electronic prescribing records and case-note review.

We generated a series of interventions that we tested by completing three Plan, Do, Study, Act (PDSA) cycles over the 3-year period. After each intervention, we completed a further 6-month evaluation of standards of diabetes care as described in the baseline audit. After each PDSA cycle, results were presented to the 'Sign up to Safety' working group where interventions were formally evaluated. Lessons were shared and discussions involving all group members informed the next stage of the quality improvement cycle, which would support the trust diabetes perioperative strategy. We absolutely recognised the importance of formal, structured meetings as opposed to opportunistic discussions to help encourage engagement and facilitate improvement in practice.

\section{STRATEGY}

The project's aim was to reduce hypoglycaemia, medication management errors and patient harm events in vascular patients by $50 \%$ over a 3 -year period. We undertook three PDSA test cycles. Each cycle was 6 months in length and all patients with diabetes undergoing major LLA were included. Patient demographics were similar between each cycle.

PDSA cycle 1 (2015) n=15: Our initial intervention was to provide clear guidelines for diabetes care and diabetes specialist nurse referral on surgical wards. These were incorporated into the departmental handbook and published on the intranet. Junior doctors were provided with a hard copy of the guidelines on induction and diabetes care was specifically included in their induction 


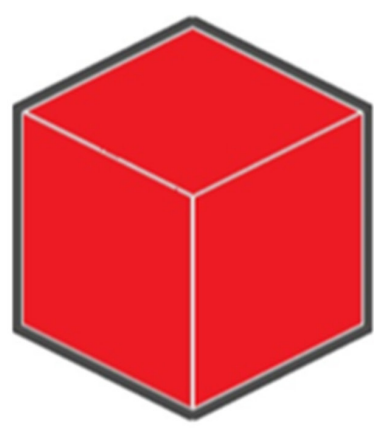

\section{Red sugar cube $=$ Action required}

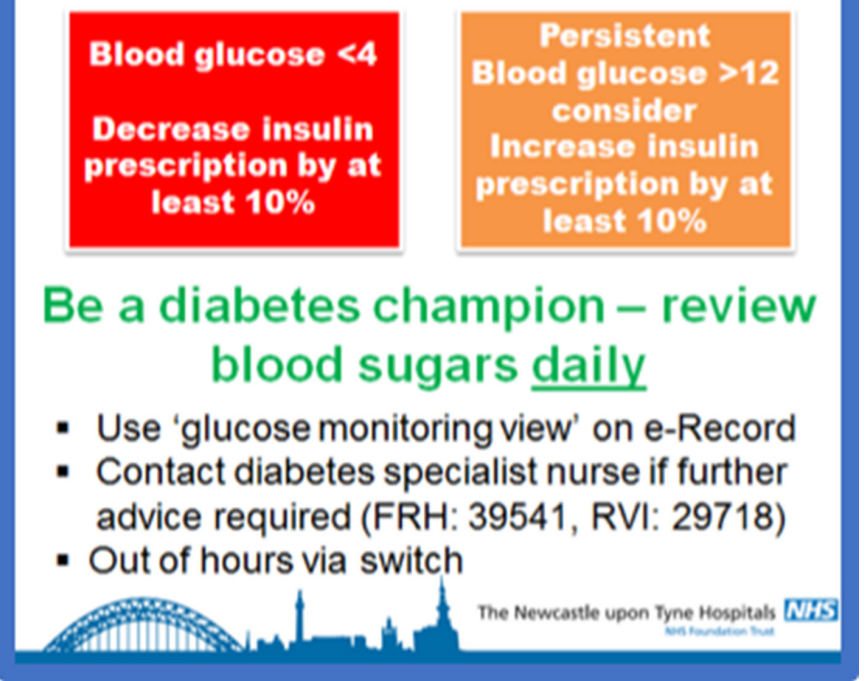

Figure 1 Poster promoting intended actions for sugar cube whiteboard alert system. FRH, Freeman Hospital; RVI, Royal Victoria Infirmary.

package. A mandatory e-learning package of safe insulin prescribing was introduced and $100 \%$ of doctors working on the vascular ward had completed this training. Monthly diabetes education meetings were implemented, attended by members of the ward multidisciplinary team (MDT). Key topics included basic glycaemic control, insulin prescribing, management of hypoglycaemia and diabetes emergencies. Additional teaching was provided to the ward nursing staff by the diabetes specialist team.

Although the guidelines and educational strategy were very positively received, there was little impact on standards of diabetes care provided. Levels of adequate diabetes monitoring improved to $82 \%$ on the surgical ward, but hypoglycaemia rates remained high and patient harm events increased. Diabetes specialist input remained low at $20 \%$ of appropriate patients. Concerns were raised about the lack of face-to-face leadership on surgical wards to promote safe diabetes care and inadequate levels of diabetes specialist input.

PDSA cycle 2 (2016) $n=14$ : Our second cycle involved establishing a daily diabetes specialist nurse in-reach service to the vascular ward to provide daily face-to-face leadership for medical and nursing staff to promote safe and effective diabetes care. Patients with diabetes with poor glycaemic control were identified to the diabetes specialist nurse by ward nursing and medical staff to enable effective management based on specialist advice. The impact of the specialist diabetes in-reach service was evaluated over a 6-month period. Despite further improvements in diabetes monitoring to $96 \%$ and improved diabetes specialist nurse input, hypoglycaemia rates and medication errors continued to rise. The problem seemed to be at the basic level of the ward connection between abnormal blood glucose measurement and taking appropriate action, whether altering medication to improve poor glycaemic control or positively identifying patients with poor glycaemic control to the diabetes nurses for specialist support.

PDSA cycle 3 (2017) n=16: Our third cycle focused on the ward connection-positively identifying those patients with poor glycaemic control and ensuring appropriate action was taken to manage hypoglycaemia and persistent hyperglycaemia. A highly visible red sugar cube prompting mechanism was implemented to readily identify any patients who were experiencing hypoglycaemia or persistent hyperglycaemia. When a blood glucose measurement was outside the acceptable range, the responsible nurse was informed and a red sugar cube placed next to the patient's name on the ward whiteboard by the central nursing station. Intended actions were clearly defined in a poster campaign used to promote the red sugar cube intervention, with the emphasis on medication management and involvement of the diabetes team (figure 1).

The red sugar cubes remained on the white board until the senior ward sister was satisfied that glycaemic control had been reviewed, appropriate changes to diabetes medication had been made and appropriate patients had been referred to the diabetes specialist nurse team according to ward guidelines. The red sugar cube prompts were incorporated into daily morning board rounds attended by all members of the ward MDT and were readily visible to the in-reach diabetes nurse service, maintained through cycle 3. Education in regard to intended actions was provided to current and all new-starting ward staff.

\section{RESULTS}

Key data are summarised in figure 2. Each intervention cycle had a positive impact on standards of adequate diabetes monitoring with improvement from $75 \%$ in the baseline audit to $98 \%$ at the end of PDSA 3. Diabetes specialist input was $15 \%$ at the baseline audit and improved to $20 \%$ at the end of PDSA 1. The biggest improvement was seen after PDSA 2 where the diabetes nurse in-reach service was introduced and $71 \%$ of patients were reviewed during their admission. However, this figure dropped to $50 \%$ after the next cycle. Hypoglycaemia rates were initially 312 episodes per 1000 diabetes days. The hypoglycaemia rates marginally dropped to 302 per 1000 diabetes days at end of PDSA 1 but reached the highest levels of 339 per 1000 diabetes days at end of 


\begin{tabular}{|c|c|c|c|c|}
\hline Standard & Baseline & PDSA 1 & PDSA 2 & PDSA 3 \\
\hline Adequate monitoring & 2014 & 2015 & 2016 & 2017 \\
\hline Diabetes specialist input & $75 \%$ & $82 \%$ & $96 \%$ & $98 \%$ \\
\hline $\begin{array}{c}\text { Hypoglycaemia per 1000 insulin days } \\
\text { Hypoglycaemia in insulin patients }\end{array}$ & $15 \%$ & $20 \%$ & $71 \%$ & $50 \%$ \\
\hline $\begin{array}{c}\text { management errors } \\
\text { Severe hypo management errors }\end{array}$ & $-80 \%$ & $67 \%$ & $67 \%$ & 157 \\
\hline Patient harm events & $20 \%$ & $33 \%$ & $20 \%$ & $6 \%$ \\
\hline
\end{tabular}

Figure 2 Table summarising project results. PDSA, Plan, Do, Study, Act.

PDSA 2 despite the implementation of diabetes specialist in-reach service. Introduction of the red sugar cube prompting mechanism, while continuing educational and diabetes specialist nurse in-reach initiatives, reduced hypoglycaemia rates by more than $50 \%$ following completion of PDSA 3. Insulin management errors after a hypoglycaemia were evident in $80 \%$ of cases, and this figure improved to $67 \%$ following PDSA 1 . The error rate stubbornly remained the same after the next intervention. A substantial decrease to $20 \%$ was seen after PDSA 3, which was an overall improvement of $70 \%$ from baseline audit. Insulin errors in association with severe hypoglycaemia were abolished. Patient harm events decreased from $20 \%$ to $6 \%$ over 3 years.

\section{LESSONS AND LIMITATIONS}

The combined educational and guideline intervention from PDSA 1 was not effective in reducing hypoglycaemia rates or patient harm events for patients with diabetes. Diabetes specialist nurse input was highest during the PDSA 2 cycle, but hypoglycaemia rates continued to rise and there was no overall improvement in insulin management errors. The overall aims of this project were achieved in PDSA cycle 3. Introduction of the red sugar cube prompting mechanism while continuing educational and diabetes specialist nurse in-reach initiatives reduced hypoglycaemia rates by more than $50 \%$. Overall insulin management errors for hypoglycaemia were reduced by $70 \%$ and insulin errors in association with severe hypoglycaemia were abolished, with a $75 \%$ reduction in patient harm events.

Educational initiatives and guidelines are an essential foundation to improve clinical practice but were not effective in isolation in achieving the aims of our project. Medical and nursing staff displayed increasing knowledge of optimal medication management for poor glycaemic control in educational settings, but in practice, appropriate actions were seldom taken.

Our study reiterates the importance of having regular meetings with key stakeholders that form the steering group. It was through these meetings that we were able to analyse our results and formulate a shared plan on how to proceed. We suggest this is a vital element of successful quality improvement.

We had assumed that increasing diabetes specialist input would improve outcomes and were surprised by the lack of impact of the daily in-reach specialist diabetes service on hypoglycaemia rates, medication errors and patient harm events. On reflection, we felt ward staff may have been falsely reassured by the daily presence of the diabetes specialist nurse team, with the impression that all patients with diabetes were receiving high standards of care. In reality, only patients positively identified by ward staff to the diabetes team were being actively reviewed. Opportunities to improve diabetes care by involvement of the diabetes nurses for appropriate patients were being missed. The second cycle intervention was limited by unreliable identification of patients with poor glycaemic control by the ward and specialist diabetes team.

The most effective intervention was a simple but powerful visual red sugar cube prompting system, readily identifying patients with diabetes with poor glycaemic control to ward staff and the diabetes nurse team. Intended actions were clearly defined with a focus on diabetes medication management, aiming to reduce the risk of medication errors and subsequent patient harm events. Strong clinical leadership was provided throughout the project by the senior ward sister and vascular consultants to promote intended actions.

We realised the effectiveness of the red sugar cube whiteboard prompt intervention could be impacted on by human factors in the longer term as it relied on the red sugar cubes being reliably noted on the whiteboard by ward nursing staff. This limitation has since been overcome by incorporating electronic sugar cube alerts into a new electronic whiteboard system rolled out across our trust. Sugar cube alerts are automatically generated for hypoglycaemia and hyperglycaemia on the electronic whiteboard as soon as patient blood glucose measurements are out of range, providing a sustainable and reliable prompting system.

The next step of this project is to evaluate the effect of the electronic sugar alert system at trust wide level. It would be interesting to see if this computerised, yet more passive, approach will be as effective as the manual 
version described in this project. We believe an important factor in the success of the sugar cube alert was the change in culture on the vascular surgery ward towards diabetes. There was strong leadership by example from the ward manager and consultant vascular surgeon, and this promoted the importance of acting on the alerts. With heavier use of computerised clinical systems, and increasing incorporation of integrated pop-ups, there is risk of alert fatigue. Overloading the user with numerous pop-up alerts can actually be counterproductive to good clinical practice. ${ }^{12}$ The further work planned for this project will look to investigate these factors.

In this project, we did not formally record or investigate the potential effect of patient comorbidities on results. Chronic kidney disease is a highly prevalent microvascular complication of diabetes mellitus. Insulin is predominantly renally excreted and patients with reduced glomerular filtration rate often have lower insulin requirements. ${ }^{13}$ We recognise that any work investigating hypoglycaemia in patients with diabetes on insulin should consider presence and severity of chronic kidney disease, and we plan to include this analysis in the next stage of quality improvement work.

\section{CONCLUSION}

Hypoglycaemia rates, medication management errors and patient harm events were successfully reduced in our unit using a combined strategy of guidelines, educational initiatives, a diabetes nurse in-reach service and a simple whiteboard alert system for poor glycaemic control. The whiteboard alert system provided the final key to achieving safer and more effective diabetes care and has now been adopted throughout our trust to promote safer care for all inpatients with diabetes. Promotion and clinical engagement with the whiteboard alert system is essential to achieve intended behaviours.

Strong clinical leadership by senior nursing and medical staff was required throughout the project to support a change in diabetes culture. High-quality diabetes care is now recognised as a vital element in the management of vascular surgery patients in our unit, rather than a secondary or overlooked element of perioperative ward care. In summary, a multifaceted approach is required to improve quality of diabetes care and reduce patient harm events on surgical wards.
Twitter@AminderASingh

Contributors AS designed and led PDSA 2 and 3, and wrote, edited and submitted the manuscript. AA was the ward manager and was involved in the implementation of all PDSA cycles. ED designed and led PDSA 1. LJ designed and led PDSA 1. BD designed, collected and analysed data for PDSA 2 and designed and collected data for PDSA 3. LW was senior author and guarantor throughout project and edited the manuscript.

Funding The authors have not declared a specific grant for this research from any funding agency in the public, commercial or not-for-profit sectors.

Competing interests None declared.

Patient consent Not required.

Provenance and peer review Not commissioned; externally peer reviewed.

Open access This is an open access article distributed in accordance with the Creative Commons Attribution Non Commercial (CC BY-NC 4.0) license, which permits others to distribute, remix, adapt, build upon this work non-commercially, and license their derivative works on different terms, provided the original work is properly cited, appropriate credit is given, any changes made indicated, and the use is non-commercial. See: http://creativecommons.org/licenses/by-nc/4.0/.

\section{REFERENCES}

1. NHS Digital. National Diabetes Inpatient Audit (NaDIA). 2017. https:// files.digital.nhs.uk/pdf/s/7/nadia-17-rep.pdf

2. Pecoraro RE, Reiber GE, Burgess EM. Pathways to diabetic limb amputation. Basis for prevention. Diabetes Care 1990;13:513-21.

3. Collin C, Collin J. Mobility after lower-limb amputation. Br J Surg 1995;82:1010-1.

4. Diabetes UK. Diabetes in the UK 2010: key statistics on diabetes. 2012. http://www.diabetes.org.uk/Documents/Reports/Diabetes-inthe-UK-2012.pdf

5. Dhatariya K, Levy N, Kilvert A, et al. NHS Diabetes guideline for the perioperative management of the adult patient with diabetes. Diabet Med 2012;29:420-33.

6. National Confidential Enquiry into Patient Outcome and Death. Lower Limb Amputation: Working Together. 2014. http://www.ncepod.org. uk/2014lla.htm

7. Frisch A, Chandra P, Smiley D, et al. Prevalence and clinical outcome of hyperglycemia in the perioperative period in noncardiac surgery. Diabetes Care 2010;33:1783-8.

8. Jhanji S, Thomas B, Ely A, et al. Mortality and utilisation of critical care resources amongst high-risk surgical patients in a large NHS trust. Anaesthesia 2008;63:695-700.

9. Pearse RM, Harrison DA, James $P$, et al. Identification and characterisation of the high-risk surgical population in the United Kingdom. Crit Care 2006;10:643-9.

10. George JT, Warriner D, McGrane DJ, et al. Lack of confidence among trainee doctors in the management of diabetes: the Trainees Own Perception of Delivery of Care (TOPDOC) Diabetes Study. QJM 2011;104:761-6.

11. Kwon S, Thompson R, Dellinger P, et al. Importance of perioperative glycemic control in general surgery: a report from the Surgical Care and Outcomes Assessment Program. Ann Surg 2013;257:8-14.

12. Backman R, Bayliss S, Moore D, et al. Clinical reminder alert fatigue in healthcare: a systematic literature review protocol using qualitative evidence. Syst Rev 2017;6:255.

13. Biesenbach G, Raml A, Schmekal B, et al. Decreased insulin requirement in relation to GFR in nephropathic type 1 and insulintreated type 2 diabetic patients. Diabet Med 2003;20:642-5. 